\title{
The relationship between pre-procedural elevated arterial lactate and contrast-induced nephropathy following primary percutaneous coronary intervention
}

\author{
Jun-Qing Yang ${ }^{1,2 \#}$, Xiao-Sheng Guo ${ }^{3 \#}$, Peng $\operatorname{Ran}^{2}$, Xiang-Ming Hu ${ }^{1,2}$, Ning Tan ${ }^{1,2}$ \\ ${ }^{1}$ The Second School of Clinical Medicine, Southern Medical University, Guangzhou, China; ${ }^{2}$ Department of Cardiology, Guangdong Cardiovascular \\ Institute, Guangdong Provincial Key Laboratory of Coronary Heart Disease, Guangdong Provincial People’s Hospital, Guangdong Academy of \\ Medical Sciences, Guangzhou, China; ${ }^{3}$ Department of Intensive Care Unit, Zhuhai Golden Bay Center Hospital, Guangdong Provincial People's \\ Hospital Zhuhai Hospital, Zhuhai, China \\ Contributions: (I) Conception and design: N Tan; (II) Administrative support: JQ Yang, N Tan; (III) Provision of study materials or patients: JQ Yang, \\ XS Guo, P Ran; (IV) Collection and assembly of data: JQ Yang, XS Guo, P Ran, XM Hu; (V) Data analysis and interpretation: JQ Yang, XS Guo, \\ XM Hu; (VI) Manuscript writing: All authors; (VII) Final approval of manuscript: All authors. \\ \#These authors contributed equally to this work as co-first authors. \\ Correspondence to: Ning Tan. The Second School of Clinical Medicine, Southern Medical University, Guangzhou 510515, China; Department \\ of Cardiology, Guangdong Cardiovascular Institute, Guangdong Provincial Key Laboratory of Coronary Heart Disease, Guangdong Provincial \\ People’s Hospital, Guangdong Academy of Medical Sciences, Guangzhou 510080, China. Email: gdghtanning@163.com.
}

Background: Risk stratification has been one of the main steps in preventing contrast-induced nephropathy (CIN), which is a common complication after percutaneous coronary intervention (PCI). Elevated arterial lactate is a biomarker indicating severe disease condition and post-intervention complications. The relationship between lactate and CIN has not been established. This study is performed to investigate the relationship between elevated arterial lactate level and contrast-induced nephropathy (CIN).

Methods: Patients diagnosed with ST-segment elevated myocardial infarction (STEMI) were prospectively enrolled, with lactate measured within $0.5-1$ hours before primary percutaneous coronary intervention (PCI). Patients with cardiopulmonary resuscitation, any forms of severe anaerobic condition, or end-stage renal disease undergoing dialysis were excluded. CIN was defined as an increase in serum creatinine $\geq 0.5 \mathrm{mg} / \mathrm{dL}$ or $25 \%$ within 72 hours after PCI. The Mehran Risk Score (MRS) is widely regarded as a classic risk model for CIN and the risk factors of MRS were applied in our multivariate regression analysis.

Results: Of the 227 enrolled patients, 47 (20.7\%) developed CIN according to the definition. The mean lactate level was higher in the CIN group than in the non-CIN group $(2.68 \pm 2.27$ vs. $1.74 \pm 1.94, \mathrm{P}<0.001)$. The arterial lactate level $\geq 2.0 \mathrm{mmol} / \mathrm{L}$ had $57.5 \%$ sensitivity and $75.6 \%$ specificity in predicting CIN.

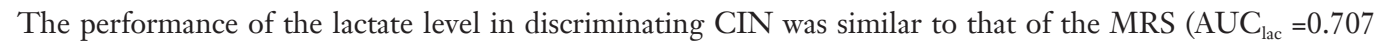
vs. $\mathrm{AUC}_{\mathrm{MRS}}=0.697, \mathrm{P}=0.86$ ). After adjusting for other risk factors, lactate $\geq 2.0 \mathrm{mmol} / \mathrm{L}$ still significantly predicted CIN (odds ratio $=3.77,95 \% \mathrm{CI}, 1.77-7.99, \mathrm{P}=0.001$ ).

Conclusions: An arterial lactate level of $\geq 2.0 \mathrm{mmol} / \mathrm{L}$ is associated with $\mathrm{CIN}$ in STEMI patients after primary PCI.

Keywords: ST-segment elevated myocardial infarction (STEMI); contrast-induced nephropathy (CIN); lactate; Mehran Risk Score (MRS); percutaneous coronary intervention (PCI)

Submitted Jul 01, 2021. Accepted for publication Aug 05, 2021.

doi: $10.21037 /$ jtd-21-1153

View this article at: https://dx.doi.org/10.21037/jtd-21-1153

(C) Journal of Thoracic Disease. All rights reserved. 


\section{Introduction}

Contrast-induced nephropathy (CIN) is the third leading cause of hospital-acquired renal failure, and increases mortality as well as the cost of health care $(1,2)$. Patients undergoing emergent percutaneous coronary intervention (PCI) had a higher risk of CIN than those undergoing elective PCI (3-5). Early identification of CIN allows for timely therapeutic interventions, such as aggressive hydration or statins, in these high risk patients undergoing emergent PCI (6-8).

Risk stratification has been one of the main steps in preventing CIN (9). Mehran et al. identified 8 variables to form the Mehran Risk Score (MRS) for predicting CIN in elective PCI (10), and it performed well in emergent PCI (11). However, the MRS includes 8 variables and the scoring system is complicated for clinical application. A systemic review showed that existing prediction models had various internally (C statistic 0.61-0.95) and externally (C statistic 0.57-0.86) discrimination value for CIN (12). On the other hand, timely coronary reperfusion is a primary consideration for STEMI patients while risk models are not widely used in this setting. A few studies have confirmed that neutrophil gelatinase-associated lipocalin and Cystatin C were promising biomarkers for predicting CIN $(13,14)$. But these new biomarkers above were not available in our center. Thus, there is a need for simple and efficient biomarker that identify patients at risk of CIN.

Lactic acid is produced through anaerobic glycolysis under hypoxic conditions, and the lactate level is widely acknowledged as an indicator of tissue perfusion status (15). Seeliger et al. reported that renal blood flow decrease and renal hypoxia had an important role in CIN pathophysiology (16). Contrast media (CM) had a direct toxicity causing vasoconstriction of the renal artery and produces ischemic injury of the kidney (6). A previous study including cardiac arrest (CA) patients revealed that high blood lactate was associated with acute kidney injury (AKI). But the blood lactate was tested after CA occurrence, so it was unclear whether AKI was caused by higher blood lactate or they were just relevant to the status of CA. Thus, blood lactate tested before procedure and cardiac event could preferably represent the baseline status (17). Therefore, this study was conducted to investigate the association of the pre-procedural arterial lactate level and rate of CIN in this high risk patients diagnosed STEMI.

We present the following article in accordance with the STARD reporting checklist (available at https://dx.doi. org/10.21037/jtd-21-1153).

\section{Methods}

\section{Study design and population}

This prospective observational study was conducted at Guangdong Cardiovascular Institute, Guangdong Provincial People's Hospital from March 2014 to August 2015. ST-segment elevated myocardial infarction (STEMI) was diagnosed according to 2013 American Heart Association (AHA)/American College of Cardiology (ACC) STEMI guideline (18). A patient exhibited with chest pain for $>30$ minute with ST-segment elevated $>2 \mathrm{~mm}$ in at least 2 contiguous pericardial electrocardiography (ECG) leads or a new left bundle branch block and arrived in our hospital within 12 or 24 hours with persistent chest pain were screening for enrollment. Exclusion criteria were as follows: (I) severe anaerobic condition including cardiopulmonary resuscitation, and any other forms of shock but not cardiopulmonary shock and thrombus (19-21); (II) lack of pre-procedural arterial lactate results before primary PCI; (III) end-stage renal disease and undergoing dialysis. All procedures performed in this study involving human participants were in accordance with the Declaration of Helsinki (as revised in 2013). The study was approved by the Guangdong Provincial People's Hospital ethical committee, and all patients provided informed consent.

\section{PCI procedure and follow-up}

All patients who met the enrollment criteria were transferred to the catheterization laboratory from the emergency department. Primary PCI was performed by expert interventional cardiologists using standard guide catheters, guidewires, balloon catheters, aspiration catheters, and stents via the radial or femoral approach. Arterial blood samples were regularly taken from the femoral or radial artery using an injection syringe full of heparin at hospital presentation or from arterial catheters before primary PCI. The sample was measured as soon as it was taken. All patients were orally administered with $300 \mathrm{mg}$ of aspirin and $300 \mathrm{mg}$ of clopidogrel as early as possible before primary PCI. The type (iodixanol, iopromide, or iopamidol) and volume of $\mathrm{CM}$ were recorded at the end of the procedure. The peri-procedural medications were left to the discretion of the operator.

All patients were referred to the clinic office or received a phone call if they were not available to complete a 30-day follow up. The composite major adverse cardiac events (MACEs) included cardiovascular death, nonfatal 
recurrent myocardial infarction (MI), stroke, acute kidney injury (AKI) requiring dialysis, and acute heart failure.

\section{Laboratory determinations, definitions and endpoints}

Arterial blood gas was measured on the GEM Premier 3000 (Hartwell Avenue Lexington, MA, USA) with a minimum detection of $0.1 \mathrm{mmol} / \mathrm{L}$. The lactate level was recorded by physician assistants who were blinded to the study. The other laboratory tests were performed according to the institute protocol.

The primary endpoint was CIN defined as an increase in serum creatinine concentration of $\geq 0.5 \mathrm{mg} / \mathrm{dL}$ or $\geq 25 \%$ from baseline within 72 hours after intravascular CM administration.

The estimated glomerular filtration rate (eGFR) was calculated with the Levy-modified Modification of Diet in Renal Disease study equation: $186.3 \times \mathrm{sCr}^{-1.154} \times$ (age in years) ${ }^{-0.208} \times 1.212$ (if the patient was black) $\times 0.742$ (if the patient was female) (22).

Anemia was defined as baseline hemoglobin $<130 \mathrm{~g} / \mathrm{L}$ for men or $120 \mathrm{~g} / \mathrm{L}$ for women (23).

Diabetes mellitus (DM) was defined according to the ADA guidelines for diabetes or recorded from the medical history of the patient (24).

Hypotension was defined as systolic blood pressure lower than $80 \mathrm{mmHg}$ for at least 1 hour or the need for inotropic medication.

MRS was regarded as the most classical risk model and was confirmed to predict CIN after primary PCI in previous studies. The risk factors of MRS would also be evaluated in our study. The MRS risk model had 8 variables including hypotension, intra-aortic balloon pump (IABP), congestive heart failure, eGFR $<60 \mathrm{~mL} / \mathrm{min} / 1.73 \mathrm{~m}^{2}$, DM, age $>75$ years, anemia, and contrast volume. The MRS was calculated by adding the score of risk factors above in each patient.

\section{Statistical analysis}

All of the analyses were performed with Stata 15.0 and R (version 3.6.3). Baseline characteristics were compared between CIN and non-CIN groups. Continuous data were presented as mean \pm standard deviation when normally distributed. Categorical variables were presented as an absolute value and percentage. The Student's $t$-test and Mann-Whitney U test were used for comparing normally distributed continuous variables and non-normally distributed continuous variables, respectively. Categorical variables were compared using the Pearson $\chi^{2}$ test or Fisher's exact test. Receiver operating characteristic (ROC) curve analysis was conducted to evaluate the performance of lactate levels and MRS. The Youden index was applied to find the best cut-off value. The areas under the curve (AUC) of the 2 correlated ROC curves were compared using a nonparametric approach (25). The multivariate logistic regression analysis was performed with a stepwise method to determine the risk factors for CIN which was a binary event variable. The Kaplan-Meier curve method was used to compare the 30-day MACE between 2 groups divided by cut-off value of lactate. The comparison was performed using the log-rank test. A two-sided $\mathrm{P}<0.05$ was considered significant.

\section{Results}

A total of 318 patients met the inclusion criteria, and 91 of them were excluded according to the exclusion criteria or loss to follow up. Finally, a total of 227 patients were enrolled in the present study. The population included 182 males $(80.2 \%)$ with a mean age of 62.6 years.

Serum creatinine measurements were available for $90.1 \%$ of patients on day 1 post-PCI, $92.9 \%$ on day 2 , and $79.3 \%$ on day 3. A total of 47 (20.7\%) patients developed CIN according to the definition of CIN.

\section{Baseline characteristics}

The baseline characteristics are listed in Table 1. Patients with CIN were more likely to be older and had a higher MRS, lactate level $(2.68 \pm 2.27$ vs. $1.74 \pm 1.94, \mathrm{P}<0.001)$, and hydration volume. Previous MI, hypotension, and use of intravenous diuretics were more frequent in the CIN group. The levels of hematocrit, eGFR, and left ventricular ejection fraction (LVEF) were significantly lower in the CIN group. The length of stents and contrast volume were similar between the 2 groups. The proportions of males, $\mathrm{DM}$, current smoker, hyperlipidemia, anemia, types of CM, thrombus aspiration, multivessel disease, and most periprocedural medications except intravenous diuretics were not significantly different between the 2 groups.

\section{Lactate level and MRS in predicting CIN}

In the ROC analysis, the best arterial lactate cut-off point was $2.0 \mathrm{mmol} / \mathrm{L}$. Lactate $\geq 2.0 \mathrm{mmol} / \mathrm{L}$ predicted CIN with a sensitivity of $57.5 \%$ and a specificity of $75.6 \%$ $\left(\mathrm{AUC}_{\mathrm{lac}}=0.707\right)$. The best cut-off point of MRS was 9 with 
Table 1 Baseline characteristics between the CIN and no CIN groups

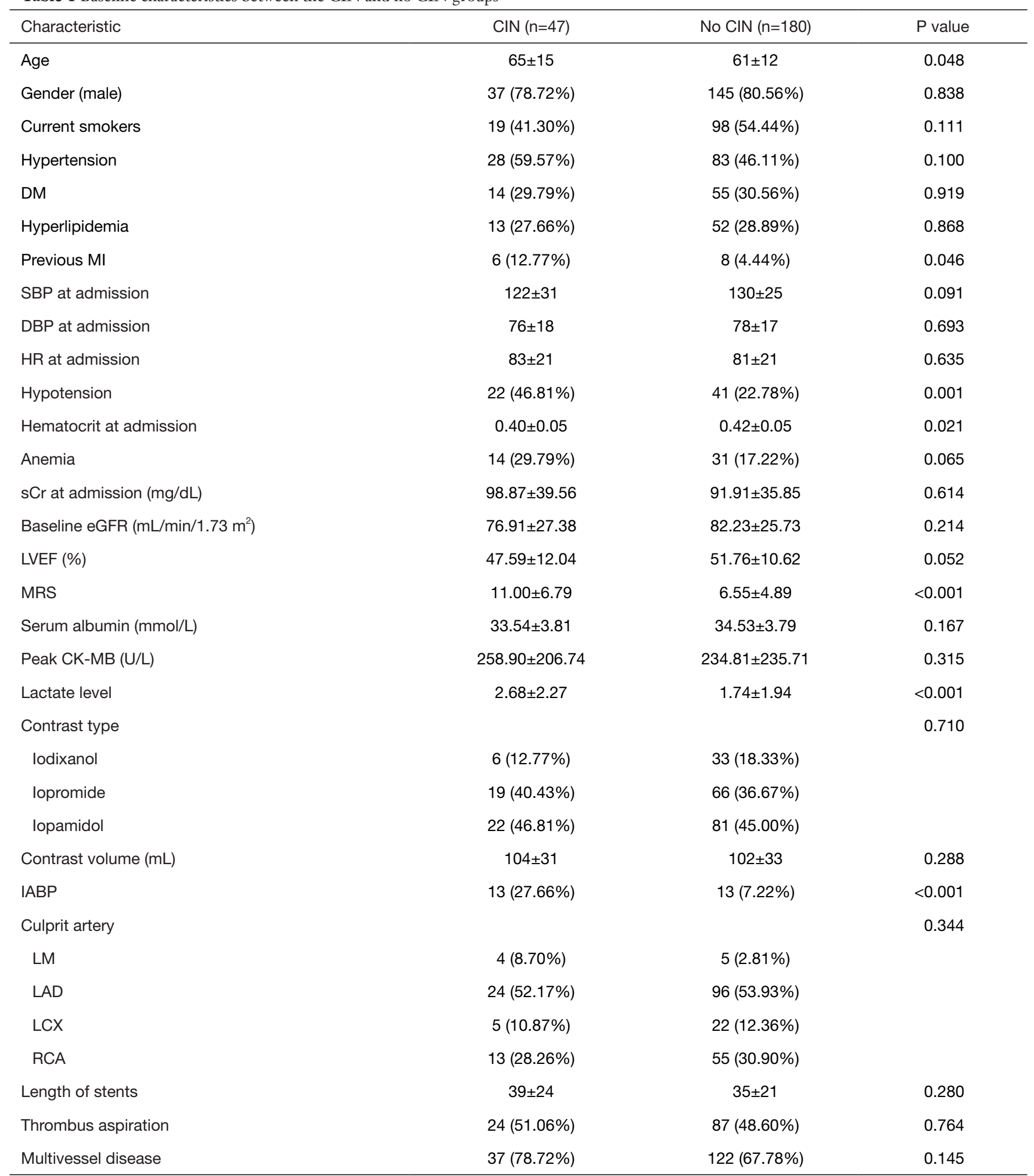

Table 1 (continued) 
Table 1 (continued)

\begin{tabular}{|c|c|c|c|}
\hline Characteristic & $\operatorname{CIN}(n=47)$ & No CIN $(n=180)$ & $P$ value \\
\hline DAPT & $44(93.62 \%)$ & $178(99.44 \%)$ & 0.029 \\
\hline ACEI/ARB & $36(76.60 \%)$ & $144(80.45 \%)$ & 0.547 \\
\hline Statins & 46 (97.87\%) & $179(100.00 \%)$ & 0.208 \\
\hline Diuretics & $36(76.60 \%)$ & $104(58.10 \%)$ & 0.020 \\
\hline Hydration volume (mL) & $1,457 \pm 650$ & $1,278 \pm 574$ & 0.044 \\
\hline
\end{tabular}

$\mathrm{CIN}$, contrast-induced nephropathy; Cl-AKI, contrast-induced acute kidney injury; DM, diabetes mellitus; MI, myocardial infarction; DBP, diastolic blood pressure; SBP, systolic blood pressure; HR, heart rate; $\mathrm{sCr}$, serum creatinine; eGFR, estimated glomerular filtration rate; LVEF, left ventricular ejection fraction; MRS, Mehran Risk Score; CK-MB, creatinine kinase MB; BNP, N-terminal pro-brain natriuretic peptide; IABP intra-aortic balloon pump; LM, left main artery; LAD, left anterior descending artery; LCX, left circumflex artery; RCA, right coronary artery; DAPT, dual antiplatelet therapy; ACEI, angiotensin converting enzyme inhibitors; ARB, angiotensin II receptor blocker.

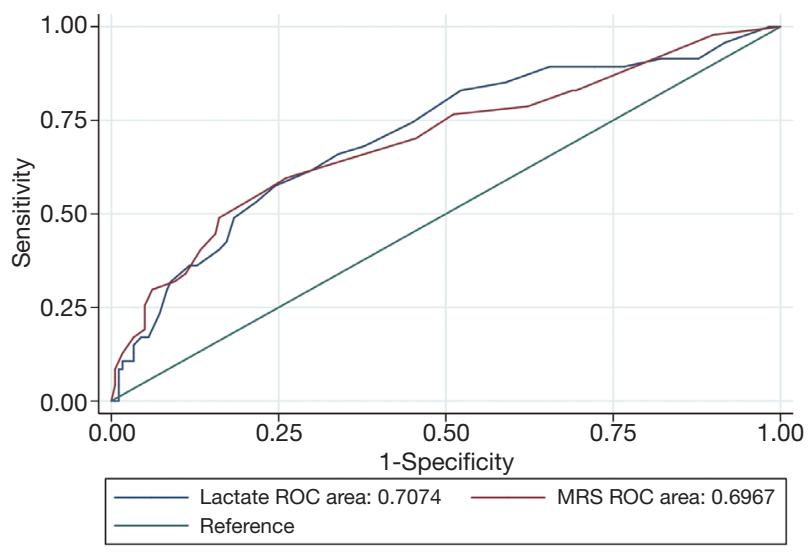

Figure 1 Receiver operating characteristic curve analysis of the arterial lactate level and MRS in the prediction of CIN. MRS, Mehran Risk Score; CIN, contrast-induced nephropathy; ROC, receiver operator characteristic.

a sensitivity of $59.6 \%$ and a specificity of $73.9 \%\left(\mathrm{AUC}_{\mathrm{lac}}\right.$ $=0.697)$. The performance of lactate was similar to that of MRS $\left(\mathrm{AUC}_{\mathrm{lac}}=0.707 v s . \mathrm{AUC}_{\mathrm{MRS}}=0.697, \mathrm{P}=0.86\right)$ (Figure 1). Nonlinear correlation analysis revealed that there was a positive smooth trend of correlation between lactate and MRS, but it was not significant (Figure 2).

\section{Association of different levels of lactate and MRS with the rate of CIN}

The population was divided into 4 groups according to

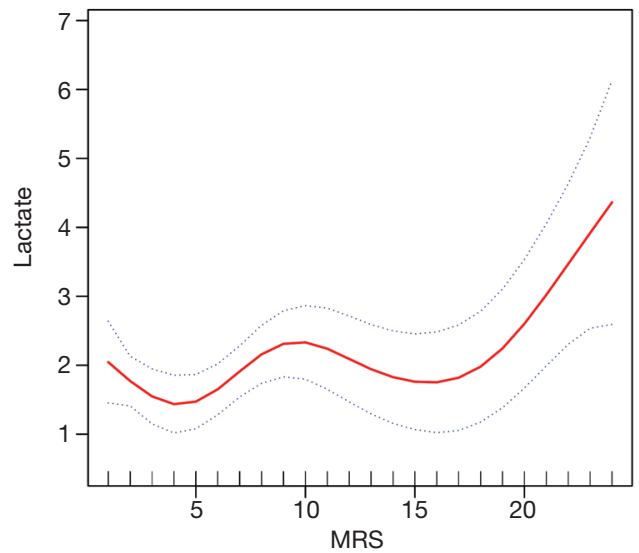

Figure 2 The nonlinear correlation analysis of lactate and MRS. MRS, Mehran Risk Score.

lactate quartiles (Q1: lac $<1.1$; Q2: $1.1 \leq$ lac $\leq 1.4$; Q3: $1.5 \leq$ lac $\leq 2.1$; Q4: lac $>2.1)$ and MRS scores (G1: MRS $\leq 5$; G2: $6 \leq \mathrm{MRS} \leq 10 ; \mathrm{G} 3: 11 \leq \mathrm{MRS} \leq 15$; G4: MRS $>15)$. Despite the definitions of CIN, different levels of lactate and MRS were associated with the development of CIN (Tables 2 and 3).

\section{Univariate and multivariate logistic regression analyses for $C I N$}

The study population was divided into 2 different lactate groups according to the ROC cut-off point. In the univariate logistic regression analysis, patients with an arterial lactate level of $\geq 2.0 \mathrm{mmol} / \mathrm{L}$ were more likely to 
Table 2 Association between lactate quartiles and the rate of CIN by different definitions

\begin{tabular}{|c|c|c|c|c|c|}
\hline CIN definitions & \multicolumn{5}{|c|}{ Outcome } \\
\hline $\mathrm{sCr}$ increase $\geq 0.5 \mathrm{mg} / \mathrm{dL}$ or $\geq 25 \%$ within $72 \mathrm{~h}, \mathrm{n}$ (\%) & $5(9.1)$ & $7(12.5)$ & $12(20.0)$ & $23(41.1)$ & $<0.001$ \\
\hline sCr increase $\geq 0.5$ mg/dL within 72 h, n (\%) & $0(0)$ & $2(3.6)$ & $1(1.7)$ & $8(14.3)$ & 0.002 \\
\hline $\mathrm{sCr}$ increase $\geq 0.3 \mathrm{mg} / \mathrm{dL}$ or $50 \%$ within $72 \mathrm{~h}, \mathrm{n}(\%)$ & $3(5.5)$ & $3(5.5)$ & $6(10.0)$ & $12(21.4)$ & 0.018 \\
\hline
\end{tabular}

$\mathrm{CIN}$, contrast-induced nephropathy; sCr, serum creatinine.

Table 3 Association between 4 groups of MRS and the rate of CIN by different definitions

\begin{tabular}{|c|c|c|c|c|c|}
\hline CIN definitions & \multicolumn{5}{|c|}{ Outcome } \\
\hline SCr increase $\geq 0.5 \mathrm{mg} / \mathrm{dL}$ or $\geq 25 \%$ within $72 \mathrm{~h}, \mathrm{n}(\%)$ & $11(11.1)$ & $13(17.1)$ & $9(33.3)$ & $14(56.0)$ & $<0.001$ \\
\hline SCr increase $\geq 0.5$ mg/dL within 72 h, n (\%) & $1(1.0)$ & $2(2.6)$ & $3(11.1)$ & $5(20.0)$ & $<0.001$ \\
\hline SCr increase $\geq 0.3 \mathrm{mg} / \mathrm{dL}$ or $50 \%$ within $72 \mathrm{~h}, \mathrm{n}(\%)$ & $3(3.1)$ & $6(7.9)$ & $6(22.2)$ & $9(36.0)$ & $<0.001$ \\
\hline
\end{tabular}

MRS, Mehran Risk Score; CIN, contrast-induced nephropathy; sCr, serum creatinine.

develop CIN compared to those with a level of $<2.0 \mathrm{mmol} / \mathrm{L}$ (odds ratio $=4.17,95 \% \mathrm{CI}, 2.13-8.16, \mathrm{P}<0.001)$. After adjusting for age $>75$ years, chronic heart failure $(\mathrm{CHF})$, $\mathrm{DM}$, anemia, eGFR $<60 \mathrm{~mL} / \mathrm{min} / 1.73 \mathrm{~m}^{2}$, CM volume $>100 \mathrm{~mL}$, hypotension, and IABP use, the arterial lactate level was still associated with CIN (odds ratio $=3.77$, 95\% CI, 1.77-7.99, $\mathrm{P}=0.001$ ) in the multivariate logistic regression analysis (Figure 3).

\section{Lactate level and outcome}

The 30-day MACEs occurred in 27 (11.9\%) of 227 patients. The Kaplan-Meier curve revealed that an arterial lactate level of $\geq 2.0 \mathrm{mmol} / \mathrm{L}$ was associated with 30-day MACEs at follow up (18.3\% vs. 9.0\%, $\mathrm{P}=0.037)$ (Figure 4).

\section{Discussion}

To the best of our knowledge, the present study is the first study to investigate the relationship between the preprocedural lactate level and CIN after primary PCI. It was demonstrated that an arterial lactate level of $\geq 2.0 \mathrm{mmol} / \mathrm{L}$ was a strong predictor of CIN in these patients. The measurement of the lactate level after primary PCI helped identify patients at high risk of developing CIN. The results showed that the performance of the lactate level was similar to that of the MRS in discriminating CIN in this population. Furthermore, the study demonstrated the prognostic value of the arterial lactate level for short-term clinical outcomes in this population.

This study found that patients with elevated arterial lactate levels were more likely to develop CIN before primary PCI. The arterial lactate level is an easily available and reproducible biomarker for predicting in-hospital complications including renal damage. Consistent with previous studies, the lactate level was related to postoperative AKI and poor outcome. Lopez-Delgado et al. demonstrated that a higher arterial lactate level 24 hours after admission was a predictor of AKI and in-hospital mortality in cardiac surgery patients (26). Zhang et al. transformed the lactate level by natural $\log (\mathrm{L} \ln )$ and found $\mathrm{L} \ln$ to be independently associated with postoperative AKI in patients undergoing cardiopulmonary bypass surgery (27). The present study confirmed that the arterial lactate level, a point-of-care measurement, could predict CIN after cardiac catheterization without transformation. Besides, the lactate levels were obtained within $0.5-1$ hours before primary PCI in this study, which helped initiate prophylactic measures in time.

Furthermore, the present study suggested that a lactate level of $\geq 2.0 \mathrm{mmol} / \mathrm{L}$ was associated with $\mathrm{CIN}$ by ROC 


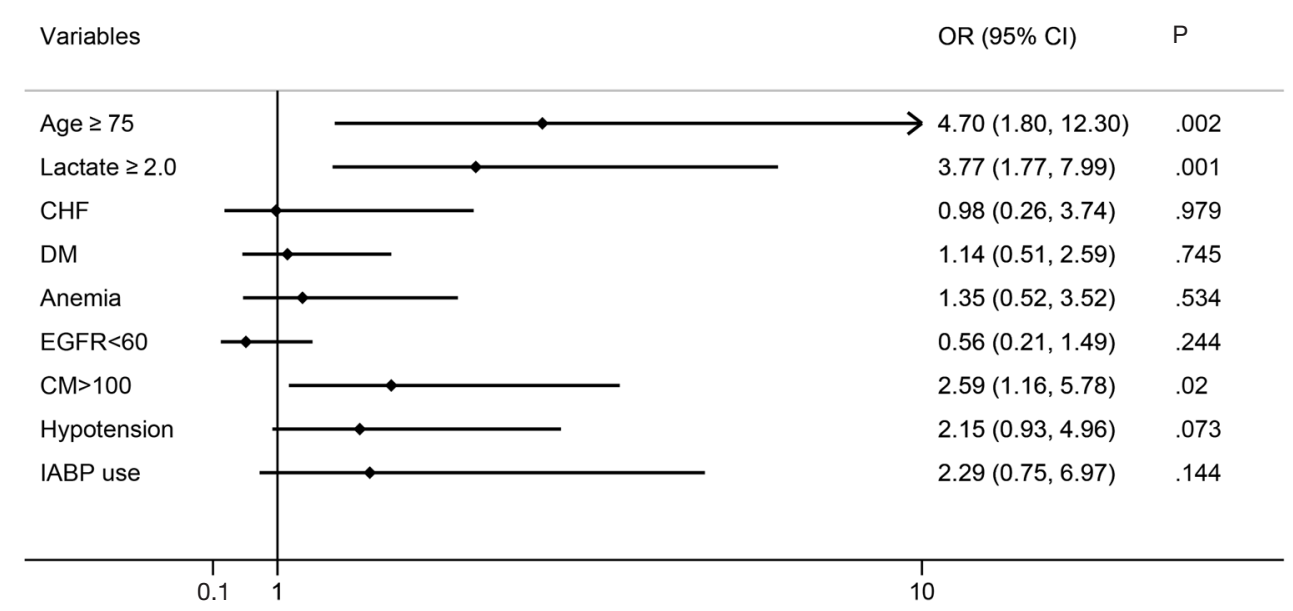

Figure 3 The multivariate logistic regression of CIN. CIN, contrast-induced nephropathy; CHF, chronic heart failure; DM, diabetes mellitus; eGFR, estimated glomerular filtration rate; CM, contrast media; IABP, intra-aortic balloon pump.

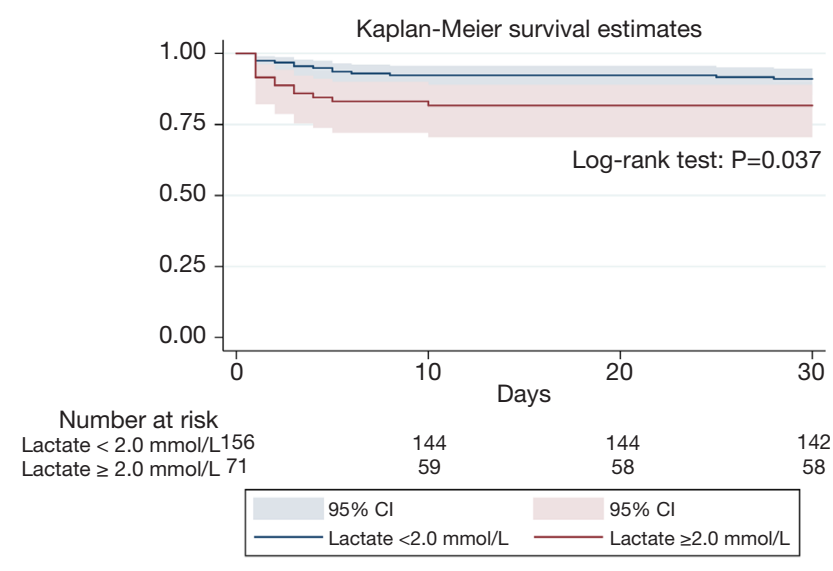

Figure 4 Kaplan-Meier curve of 30-day MACEs in patients with an arterial lactate level of $\geq 2.0$ and $<2.0 \mathrm{mmol} / \mathrm{L}$. MACE, major adverse cardiac event.

curve analysis. De Corte $e t$ al. found that severe lactic acidosis (serum lactate $>5 \mathrm{mmol} / \mathrm{L}$ and $\mathrm{pH}<7.35$ ) was more frequent in intensive care unit patients with AKI treated with renal replacement therapy (28). Vermeulen et al. found a correlation between the blood lactate level and other risk factors in patients with STEMI (29). They divided 1,176 patients into 3 groups based on the lactate tertiles and found that elevated lactate levels were related to impaired hemodynamics (hypotension, IABP) and 30-day mortality. Although the relationship between the lactate level and in-hospital complications was illustrated, the cut-off point was not well established in the aforementioned 2 studies. The present study applied ROC curve analysis to determine the cut-off point and divided the population into 2 groups based on the cut-off point. The cutoff value was confirmed by logistic regression analysis, and a lactate level of $\geq 2.0 \mathrm{mmol} / \mathrm{L}$ could predict $\mathrm{CIN}$ after adjusting for other risk factors. In clinical practice, lactate $\geq 2.0 \mathrm{mmol} / \mathrm{L}$ is also considered an abnormal value which will attract the attention of physicians. The best cut-off point of our study is consistent with present clinical practice.

This study also demonstrated that the lactate level was similar to the MRS as a discriminator for CIN. Several risk models have been applied for predicting CIN $(10,30,31)$. MRS was regarded as the most classical one among these models and was confirmed to predict CIN after primary PCI in our study (AUC =0.697). The ROC curve analysis showed that the AUC was not significantly different between the lactate level and the MRS in this study $(\mathrm{P}=0.86)$. Both the biomarker and the risk model performed well in recognizing high-risk patients. However, MRS has not been as widely used in the case of emergent PCI because some variables might not be available in patients who were treated as quick as possible with primary PCI. It is more convenient to obtain the arterial lactate results than to calculate the MRS in some cases because a blood sample can be obtained quickly and the lactate level can be tested within minutes with the point-of-care technique. Thus, effective prophylactic measures such as hydration can be decided and delivered in a timely manner to prevent CIN. This is particularly valuable in the case of primary PCI. Furthermore, lactate could still predict CIN despite the effects of 8 variables of the MRS in the multivariate logistic regression analysis (odds ratio $=3.77, \mathrm{P}=0.001$ ).

The reason why the lactate level is able to predict 
CIN after primary PCI may be related to the following pathophysiological mechanism $(1,6,16,32)$. Impaired myocardial contractility in patients with STEMI decreases the effective circulating blood volume, which cuts down the renal flow and increases the lactate level. Consequently, renal hypoperfusion is exacerbated as a result of activation of the sympathetic nervous system and the renin-angiotensinaldosterone system, which in turn decreases the renal flow. Reducing the renal flow increases the contact time between cytotoxic $\mathrm{CM}$ and renal vascular endothelial and tubular epithelial cells and accelerates the process of CIN.

The present study preliminarily verified elevated lactate levels as a risk factor for 30-day MACEs. This was an acceptable result, since the lactate level has also shown prognostic predictive value in patients with STEMI in other studies (33).

This study had several limitations. Firstly, it was a smallscale, single-center, and observational study. Hence, the conclusion needs to be confirmed through large-scale trials. Secondly, no data were available on persistent lactate levels after PCI, so the study failed to calculate the lactate clearance. Thirdly, due to the small sample size, subgroup analysis was not performed to explore the relationships in different subgroups in detail.

\section{Conclusions}

In this single-center study, the arterial lactate level was a promising biomarker for identifying CIN and poor shortterm outcomes in patients with STEMI undergoing primary PCI. The arterial lactate level may be a rapid tool for CIN risk stratification, independent of and as effective as the MRS model.

\section{Acknowledgments}

Funding: The study was supported by Medical Scientific Research Foundation of Guangdong Province, China (No. A2020428). This study was also supported by grants from Sailing Foundation (Grant No.: LHJJ201611011, LHJJ201612127). The funders had no role in the study design, data collection and analysis, decision to publish, or preparation of the article. The work was not funded by any industry sponsors.

\section{Footnote}

Reporting Checklist: The authors have completed the
STARD reporting checklist. Available at https://dx.doi. org/10.21037/jtd-21-1153

Data Sharing Statement: Available at https://dx.doi. org/10.21037/jtd-21-1153

Conflicts of Interest: All authors have completed the ICMJE uniform disclosure form (available at https://dx.doi. org/10.21037/jtd-21-1153). The authors have no conflicts of interest to declare.

Ethical Statement: The authors are accountable for all aspects of the work in ensuring that questions related to the accuracy or integrity of any part of the work are appropriately investigated and resolved. All procedures performed in this study involving human participants were in accordance with the Declaration of Helsinki (as revised in 2013). The study was approved by the Guangdong Provincial People's Hospital ethical committee [No. GDREC2014157H(R1)], and all patients provided informed consent.

Open Access Statement: This is an Open Access article distributed in accordance with the Creative Commons Attribution-NonCommercial-NoDerivs 4.0 International License (CC BY-NC-ND 4.0), which permits the noncommercial replication and distribution of the article with the strict proviso that no changes or edits are made and the original work is properly cited (including links to both the formal publication through the relevant DOI and the license). See: https://creativecommons.org/licenses/by-nc-nd/4.0/.

\section{References}

1. Chandiramani R, Cao D, Nicolas J, et al. Contrastinduced acute kidney injury. Cardiovasc Interv Ther 2020;35:209-17.

2. De Filippo O, D’Ascenzo F, Piroli F, et al. Sometimes neither water nor fire are more useful than friendshipa new risk score for prediction of contrast-induced nephropathy (CIN) and long-term adverse outcomes in patients undergoing coronary angiography. J Thorac Dis 2019;11:2675-79.

3. Tsai TT, Patel UD, Chang TI, et al. Contemporary incidence, predictors, and outcomes of acute kidney injury in patients undergoing percutaneous coronary interventions: insights from the NCDR Cath-PCI registry. JACC Cardiovasc Interv 2014;7:1-9.

4. Narula A, Mehran R, Weisz G, et al. Contrast-induced 
acute kidney injury after primary percutaneous coronary intervention: results from the HORIZONS-AMI substudy. Eur Heart J 2014;35:1533-40.

5. Shacham Y, Steinvil A, Arbel Y. Acute kidney injury among ST elevation myocardial infarction patients treated by primary percutaneous coronary intervention: a multifactorial entity. J Nephrol 2016;29:169-74.

6. Azzalini L, Spagnoli V, Ly HQ. Contrast-Induced Nephropathy: From Pathophysiology to Preventive Strategies. Can J Cardiol 2016;32:247-55.

7. Zhou X, Dai J, Xu X, et al. Comparative Efficacy of Statins for Prevention of Contrast-Induced Acute Kidney Injury in Patients With Chronic Kidney Disease: A Network Meta-Analysis. Angiology 2019;70:305-16.

8. Zuo P, Li Y, Zuo Z, et al. Glycemic variability as predictor of contrast-induced nephropathy in diabetic patients with acute myocardial infarction undergoing percutaneous coronary intervention. Ann Transl Med 2020;8:1505.

9. Waybill MM, Waybill PN. Contrast media-induced nephrotoxicity: identification of patients at risk and algorithms for prevention. J Vasc Interv Radiol 2001;12:3-9.

10. Mehran R, Aymong ED, Nikolsky E, et al. A simple risk score for prediction of contrast-induced nephropathy after percutaneous coronary intervention: development and initial validation. J Am Coll Cardiol 2004;44:1393-9.

11. Sgura FA, Bertelli L, Monopoli D, et al. Mehran contrast-induced nephropathy risk score predicts shortand long-term clinical outcomes in patients with STelevation-myocardial infarction. Circ Cardiovasc Interv 2010;3:491-8.

12. Silver SA, Shah PM, Chertow GM, et al. Risk prediction models for contrast induced nephropathy: systematic review. BMJ 2015;351:h4395.

13. Tong J, Li H, Zhang H, et al. Neutrophil Gelatinaseassociated Lipocalin in the Prediction of Contrast-induced Nephropathy: A Systemic Review and Meta-analysis. J Cardiovasc Pharmacol 2015;66:239-45.

14. D'Amore C, Nuzzo S, Briguori C. Biomarkers of Contrast-Induced Nephropathy: Which Ones are Clinically Important? Interv Cardiol Clin 2020;9:335-44.

15. Andersen LW, Mackenhauer J, Roberts JC, et al. Etiology and therapeutic approach to elevated lactate levels. Mayo Clin Proc 2013;88:1127-40.

16. Seeliger E, Sendeski M, Rihal CS, et al. Contrast-induced kidney injury: mechanisms, risk factors, and prevention. Eur Heart J 2012;33:2007-15.

17. Sandroni C, Dell'anna AM, Tujjar O, et al. Acute kidney injury after cardiac arrest: a systematic review and meta-analysis of clinical studies. Minerva Anestesiol 2016;82:989-99.

18. O'Gara PT, Kushner FG, Ascheim DD, et al. 2013 ACCF/ AHA guideline for the management of ST-elevation myocardial infarction: a report of the American College of Cardiology Foundation/American Heart Association Task Force on Practice Guidelines. J Am Coll Cardiol 2013;61:e78-e140.

19. Dettmer M, Holthaus CV, Fuller BM. The impact of serial lactate monitoring on emergency department resuscitation interventions and clinical outcomes in severe sepsis and septic shock: an observational cohort study. Shock 2015;43:55-61.

20. Shankar-Hari M, Phillips GS, Levy ML, et al. Developing a New Definition and Assessing New Clinical Criteria for Septic Shock: For the Third International Consensus Definitions for Sepsis and Septic Shock (Sepsis-3). JAMA 2016;315:775-87.

21. Maekawa K, Sugita C, Yamashita A, et al. Higher lactate and purine metabolite levels in erythrocyte-rich fresh venous thrombus: Potential markers for early deep vein thrombosis. Thromb Res 2019;177:136-44.

22. Levey AS, Bosch JP, Lewis JB, et al. A more accurate method to estimate glomerular filtration rate from serum creatinine: a new prediction equation. Modification of Diet in Renal Disease Study Group. Ann Intern Med 1999;130:461-70.

23. Nutritional anaemias. Report of a WHO scientific group. World Health Organ Tech Rep Ser 1968;405:5-37.

24. Inzucchi SE, Bergenstal RM, Buse JB, et al. Management of hyperglycemia in type 2 diabetes: a patient-centered approach: position statement of the American Diabetes Association (ADA) and the European Association for the Study of Diabetes (EASD). Diabetes Care 2012;35:1364-79.

25. DeLong ER, DeLong DM, Clarke-Pearson DL. Comparing the areas under two or more correlated receiver operating characteristic curves: a nonparametric approach. Biometrics 1988;44:837-45.

26. Lopez-Delgado JC, Esteve F, Torrado H, et al. Influence of acute kidney injury on short- and long-term outcomes in patients undergoing cardiac surgery: risk factors and prognostic value of a modified RIFLE classification. Crit Care 2013;17:R293.

27. Zhang Z, Ni H. Normalized lactate load is associated with development of acute kidney injury in patients who underwent cardiopulmonary bypass surgery. PLoS One 2015;10:e0120466. 
28. De Corte W, Vuylsteke S, De Waele JJ, et al. Severe lactic acidosis in critically ill patients with acute kidney injury treated with renal replacement therapy. J Crit Care 2014;29:650-5.

29. Vermeulen RP, Hoekstra M, Nijsten MW, et al. Clinical correlates of arterial lactate levels in patients with STsegment elevation myocardial infarction at admission: a descriptive study. Crit Care 2010;14:R164.

30. Bartholomew BA, Harjai KJ, Dukkipati S, et al. Impact of nephropathy after percutaneous coronary intervention and a method for risk stratification. Am J Cardiol 2004;93:1515-9.

31. Liu YH, Liu Y, Tan N, et al. Predictive value of GRACE risk scores for contrast-induced acute kidney injury in patients with ST-segment elevation myocardial infarction before undergoing primary percutaneous coronary intervention. Int Urol Nephrol 2014;46:417-26.

32. Tehrani S, Laing C, Yellon DM, et al. Contrast-induced acute kidney injury following PCI. Eur J Clin Invest 2013;43:483-90.

33. Lazzeri C, Valente S, Chiostri M, et al. Lactate in the acute phase of ST-elevation myocardial infarction treated with mechanical revascularization: a single-center experience. Am J Emerg Med 2012;30:92-6.

(English Language Editor: C. Betlazar-Maseh)
Cite this article as: Yang JQ, Guo XS, Ran P, Hu XM, Tan $\mathrm{N}$. The relationship between pre-procedural elevated arterial lactate and contrast-induced nephropathy following primary percutaneous coronary intervention. J Thorac Dis 2021;13(9):54675476. doi: $10.21037 / \mathrm{jtd}-21-1153$ 